\title{
Frakturen der Halswirbelsäule bei ankylosierender Spondylitis und diffuser idiopathischer skeletaler Hyperostose
}

Thomas Vordemvenne

\section{Einleitung}

Die Fraktur der Wirbelsäule bei ankylosierender Spondylitis (AS/Morbus Bechterew) oder DISH (diffuse idiopathische skeletale Hyperostose) ist für den Patienten eine fatale Entität. Sie stellt einen Indikator für ein Fortschreiten der Erkrankung dar und ist mit einer deutlich erhöhten Letalität verbunden. Die Frakturen sind komplex und häufig mit neurologischen Ausfällen verbunden.

Die ankylosierende Spondylitis zählt zum Formenkreis der chronisch rheumatischen Erkrankungen. Sie manifestiert sich hauptsächlich am Achsenskelett und führt im Krankheitsverlauf zu destruktiven und osteoplastischen Veränderungen der Wirbelsäule und der lliosakralgelenke. Typisch an der Wirbelsäule sind fortschreitende Verknöcherungen der Längsbänder und der Bandscheiben, die zu einer kompletten Einsteifung der Segmente führen - die sog. Bambuswirbelsäule. Dies geht einher mit einer vermehrten Kyphose der BWS und einer kompensatorischen Lordose der HWS bei vermehrter Kippung des Beckens. Auch osteolytische Veränderungen der Wirbelkörper können entstehen - die sog. Andersson-Läsion, die sekundär bakteriell bedingt oder primär aseptischen Hintergrundes sein kann. Durch die veränderte Lastverteilung kommt es zu einer Rarefizierung der Trabekelstruktur und zur Osteoporose der Wirbelkörper.

Die diffuse idiopathische skeletale Hyperostose (DISH) wurde von Forestier 1950 erstmals als senile vertebrale ankylosierende Hyperostose beschrieben [1]. Die Ursache ist unbekannt. Typisch sind paravertebrale und paradiskale Knochenformationen, insbesondere des vorderen Längsbandes, die sich kraniokaudal ausbreiten und funktionell - wie beim Morbus Bechterew - zu einer multisegmentalen Einsteifung der Wirbelsäule führen. Die Knochendichte ist bei der DISH eher angehoben [2]. Die osteophytären ventralen und paradiskalen Ausziehungen an der HWS können derart raumfordernd wirken, dass es bereits ohne Verletzung zu Schluckstörungen und nervalen Kompressionen kommt. Dies ist anamestisch vor operativen Eingriffen und der Wahl des Zuganges zu beachten.
Das Patientengut ist durch eine extrem erhöhte Morbidität von $50 \%$ und Mortalität von 20-30\% gekennzeichnet. $80 \%$ der Patienten haben eine schwere Komplikation infolge der Verletzung oder der Therapie [3].

Im späten Stadium der Erkrankungen ist eine Differenzierung zwischen DISH und AS rein bildmorphologisch oft nur schwer zu treffen. Beiden gemein ist gerade beim älteren Patienten das deutlich erhöhte Risiko einer Wirbelsäulenfraktur infolge eines Bagatelltraumas.

\section{Merke}

Insbesondere ältere Bechterew-/DISH-Patienten erleiden nach Bagatelltrauma eine Fraktur - die Komplikationsrate ist hoch.

\section{Diagnostik}

Aufgrund der geänderten Biomechanik der Wirbelsäule ist eine sorgsame Abklärung der gesamten Wirbelsäule sinnvoll. Diese Empfehlung wird durch die Tatsache unterstützt, dass traumatische Läsionen nach Bagatelltraumen entstehen und initial nicht unbedingt von einem deutlichen Schmerz begleitet sind. Auch werden zeitgleiche Verletzungen verschiedener Wirbelsäulenabschnitte gesehen. Dem konventionellen Röntgen entgeht häufig die Diagnose, da osteophytäre Überlagerungen, osteopene Knochenstruktur, Überlagerungen des Schultergürtels im zervikothorakalen Übergang und initial wenig dislozierte Frakturverläufe die Abbildung erschweren. 36\% der Frakturen entgehen der initialen Diagnose im Bereich der unteren HWS [4-7].

Aus diesem Grunde kann bei dieser Patientengruppe die CT-Bildgebung der gesamten Wirbelsäule - auch bei Bagatelltraumen - generell empfohlen werden. Bei neurologischen Ausfällen ist eine MRT zu ergänzen.

\section{Merke}

Ein Patient nach Sturz mit AS oder DISH ist solange als Wirbelsäulenverletzter zu behandeln, bis das Gegenteil sicher ausgeschlossen ist.

\section{Merke}

Bei der ankylosierenden Spondylitis entsteht eine osteoporotische Knochenqualität, bei der DISH ist die Knochendichte eher angehoben. 


\section{Verletzungsmuster}

Aus traumatologischer und biomechanischer Sicht ist die Rigidität der Wirbelsäule im späten Stadium beider Erkrankungen derart ausgeprägt, dass die gesamte kinetische Energie bei einem Unfallereignis mit einer maximalen Hebellast auf die eingesteiften Segmente einwirkt. Dies führt zu einem charakteristischen Verletzungsbild, welches immer bei der AS sowohl die ventralen als auch dorsalen Strukturen betrifft. Somit liegen ausnahmslos instabile Komplexfrakturen des AO-Typs B oder C vor.

Merke
Biomechanisch ist die Bechterew-Fraktur mit der
Fraktur eines langen Röhrenknochens vergleichbar.

Neurologische Defizite sind häufig. Der größte Teil der zervikalen Frakturen ist lokalisiert auf Höhe der unteren HWS und des zervikothorakalen Überganges. Der Frakturverlauf ist dabei typischerweise bei Hyperextensionsmechanismen transdiskal und/oder transkorporell in die dorsalen Strukturen auslaufend. Bei Flexionsverletzungen kann es zu ossären Kompressionsdefekten der ventralen Säule mit Zerreißung der dorsalen Strukturen kommen.

Wichtige Begleitverletzung ist eine Dissektion der A. vertebralis oder der A. carotis. Aus diesem Grunde sollte bei Frakturnachweis die Diagnostik mit einer CT- oder MRAngiografie kombiniert werden, um thrombembolische Komplikationen zu vermeiden [8]. Zudem ist der Gefäßstatus wichtig für die präoperative Planung der Schraubenpositionierung.

Der Sonderstellung der ankylosierenden Wirbelsäule wie auch dem Status der A. vertebralis wird in der aktuellen Klassifikation der AO mittels der Modifier M3 und M4 Rechnung getragen [9].

\section{Konservative Therapie}

Obgleich von wenigen Autoren die konservative Therapie bei Frakturen ohne neurologisches Defizit empfohlen wird, ist das Outcome in größeren Patientenkollektiven nicht zufriedenstellend. Die Entscheidung gegen eine operative Stabilisierung ist bei multimorbiden Patienten ohne Neurologie im Einzelfall zu rechtfertigen. Allerdings ist auch hier der Maßstab anzusetzen, die Mobilisationsfähigkeit des Patienten unter konservativer Orthesenversorgung in gleichem Maße zu gewährleisten wie im Falle einer operativen Stabilisierung. Auch muss garantiert sein, dass eine mögliche Dislokation der Fraktur zeitnah erkannt wird, um fatale neurologische Komplikationen zu vermeiden. Beides ist eigentlich nicht zu gewährleisten.
Merke

Die konservative Therapie ist im seltenen Einzelfall zu rechtfertigen - Therapie der Wahl ist die operative Stabilisierung.

\section{Operative Therapie}

Ziele der operativen Versorgung sind 1. die primär stabile, belastungssichere Instrumentation, 2 . die ausreichende Dekompression des Spinalkanals bei Neurologie und 3. die Wiederherstellung des Alignments.

Zum Erreichen dieser Ziele stehen verschiedene Versorgungkonzepte zur Diskussion $[10,11]$. Im Folgenden wird das eigene Vorgehen beschrieben:

Der primäre Zugang bei Frakturen der HWS bei Morbus Bechterew ist dorsal. Die Instrumentierung kann klassisch mittels Massa-Schrauben oder zervikalen Pedikelschrauben erfolgen. Letzteres vorzugsweise unter intraoperativer Navigation. Pedikelschrauben garantieren die sicherste Verankerung. Der posteriore Zugang ermöglicht bei Neurologie die ausreichende Dekompression des Spinalkanals.

Nachteil des posterioren Vorgehens ist die aufwendige Lagerung der Patienten. Je nach Ausmaß der Deformität ist dies eine Herausforderung und kann mittels MayfieldKlemme oder Kopfschale erfolgen. Eine Kopftieflage oder Neutrallage ist zu vermeiden, um den ausreichenden venösen Rückstrom und eine Blutungskontrolle zu gewährleisten.

\section{Merke \\ Die primär dorsale Instrumentierung bietet alle Möglichkeiten zur belastungsstabilen Versorgung und Dekompression des Spinalkanals.}

Die Länge der Instrumentierung folgt der Biomechanik der Bechterew-Wirbelsäule: Mindestens 2 Segmente über und 2 Segmente unter der Fraktur sollten instrumentiert werden. Liegt eine Verletzung der unteren HWS resp. des zervikothorakalen Überganges vor, ist die Instrumentierung in die obere BWS zu verlängern, um die erhöhte Hebellast zu neutralisieren (junktionale Zone; Fall 1/Fall 2).

\section{FALL 1}

Patient m, 67 Jahre, Sturz in häuslicher Umgebung, Nackenschmerz, initiale Diagnostik mittels konventionellem Röntgen ( $\bullet$ Abb. 1). Erweiterung der Diagnostik mittels CT der gesamten Wirbelsäule bei bekannter ankylosierender Spondylitis ( $\bullet$ Abb. 2). 


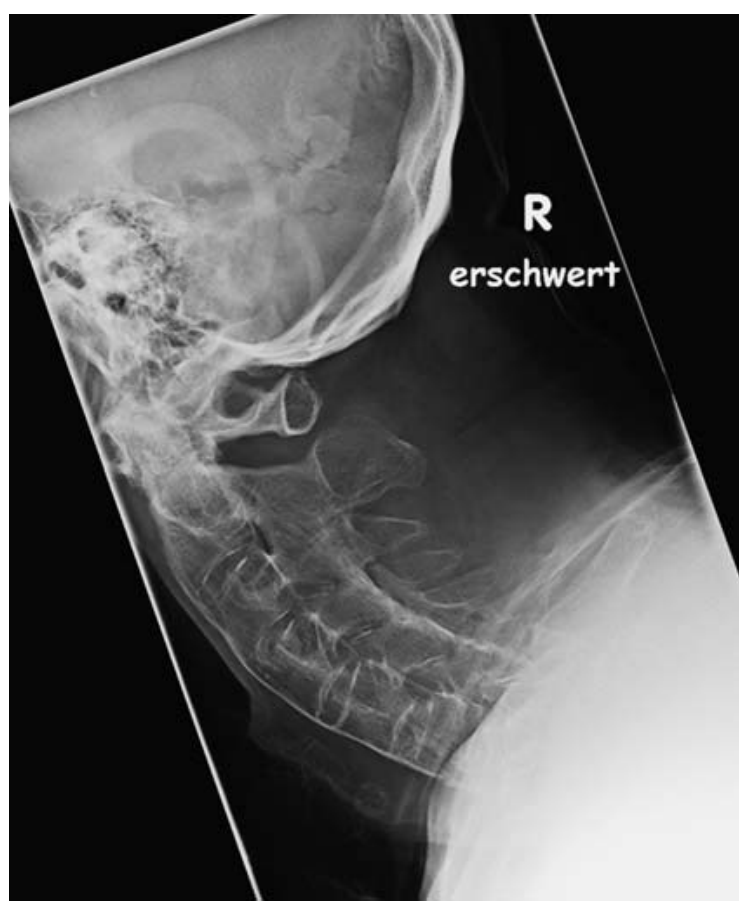

- Abb. 1 Konventionelles Röntgen eines Patienten mit Morbus Bechterew und Sturz auf ebener Erde. Keine ausreichende Abbildung von C VII. Überlagerung des Schultergürtels und osteopene Knochenstruktur - unzureichende Diagnostik zum Frakturausschluss.

\section{FALL 2}

Patient m, 79 J, Sturz auf ebener Erde, bekannter Morbus Bechterew, dislozierte transkorporelle, transdiskale Fraktur C V AO-Typ C, ASIA B, hypoplastische $A$. vertebralis rechts, dorsale Instrumentierung Massa C III/IV auf VI/VII und Dekompression, Liegezeit akut stationär 7 Tage, Verlegung in neurologische Frührehabilitation, Konsolidierung 4 Monate nach Versorgung ASIA D, CT-Nachweis der konsolidierten Fraktur ( $\triangleright$ Abb. 3).

Für die Mehrzahl der Patienten ist die dorsale, mehrsegmentale Stabilisierung ausreichend. Kommt es jedoch nach dorsaler Stabilisierung zu einem deutlichen ventralen Aufklappen durch z.B. eine reklinierende Korrektur der Achse, so muss über eine ventrale Gegenstabilisierung nachgedacht werden.

Ein primär anteriores Vorgehen als Stand-alone-Versorgung ist nicht zu empfehlen. In der Literatur finden sich Versagensraten bereits innerhalb der ersten 48 Stunden von $50 \%$ [12].

Die primäre ventrale Plattenversorgung beim Morbus Bechterew ist somit immer mit einer dorsalen Gegensta- bilisierung zu kombinieren - idealerweise innerhalb der ersten 48 Stunden.

\footnotetext{
Merke

Eine rein anteriore Plattenosteosynthese ist bei der AS nicht zu empfehlen, da die Rate des Implantatversagens inakzeptabel ist.
}

Im Falle einer Fraktur bei DISH, die isoliert die ventrale Säule betrifft und bei der eine Verletzung der dorsalen Bandstrukturen ausgeschlossen wurde, kann ein primär ventrales Vorgehen gewählt werden. Wie bereits ausgeführt, liegen bei der DISH häufig erhebliche ventrale osteophytäre Anbauten vor. Hier ist unbedingt zu beachten, diese Anbauten mittels Fräse bis auf das ursprüngliche Kortikalisniveau zu reduzieren. Bei auftragender Plattenpositionierung können ansonsten erhebliche Schluckstörungen die Folge sein (Fall 3).

FALL 3

Patient m; 86 J, Verkehrsunfall als Fußgänger, Becken-B-Verletzung, Extensionsfraktur mit transdiskaler Instabilität C III/IV AO-Typ B3, DISH, ASIA E, anteriorer Zugang, Reduktion der ventralen Osteophyten, ventrale Plattenosteosynthese, stationäre Liegezeit 3 Wochen ( $\triangleright$ Abb. 4).

\section{Merke}

Bei DISH ist die operative Abtragung der ventralen, osteophytären Anbauten essenziell, um postoperative Dysphagien durch die Platte zu vermeiden.

\section{Fazit}

Patienten mit Morbus Bechterew oder DISH haben per se eine erhöhte Morbidität und Mortalität. Kommt es zudem zu einer Fraktur der (Hals-)Wirbelsäule, ist perioperativ immer mit Komplikationen zu rechnen. Das Erkennen einer Fraktur bei AS oder DISH ist schwierig und oft verspätet. Deshalb sollte zu Beginn großzügig eine CTDiagnostik eingeleitet werden. Beachtet werden müssen begleitende Verletzungen der hirnversorgenden Gefäße.

Ein konservatives Vorgehen ist begründeten Einzelfällen ohne Neurologie vorbehalten. DISH-Patienten neigen zu Dysphagien, die sich postoperativ verstärken können.

Ziel ist die stabile Instrumentierung, um eine frühe Mobilisation zu ermöglichen. Hierbei ist die primär dorsale Stabilisierung mit Dekompression einem primär anterioren Vorgehen vorzuziehen. Eine anteriore Plattenversorgung bei AS benötigt immer und zeitnah die dorsale Gegenstabilisierung. 

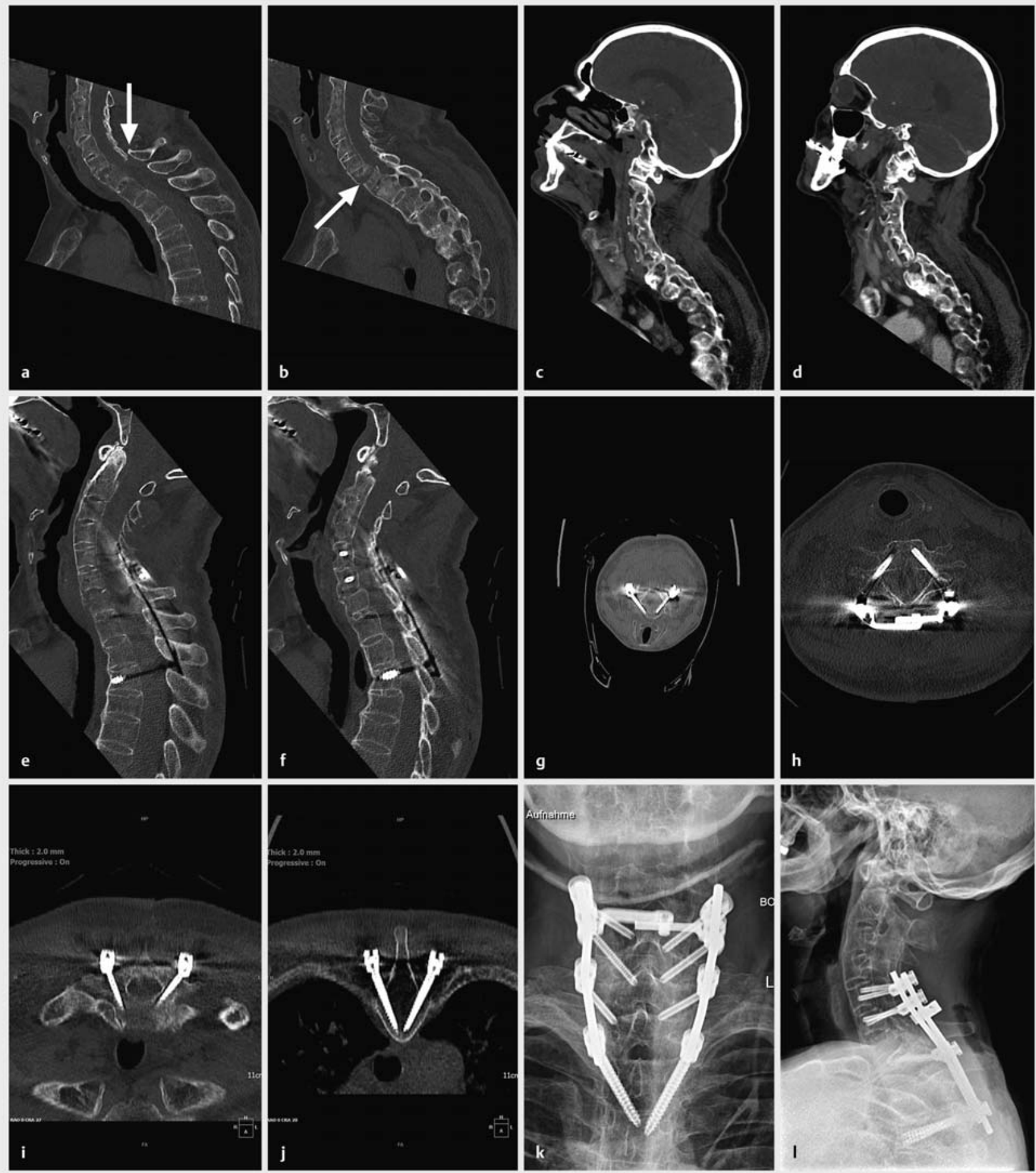

- Abb. 2 a Transkorporelle Fraktur C VII AO-Typ C mit kranialer Rotation und dorsaler Verhakung der linksseitigen Facette und Lamina, Monoverletzung, ASIA E, stationäre Liegezeit 11 Tage (Anmerkung: Pfeile zeigen Frakturdislokation). b Erweiterung der Diagnostik mittels CT-Angiografie mit Ausschluss einer Dissektion der Aa. vertebrales. c Posteriorer Zugang, offene Reposition/Enthakung und dorsale navigierte, pedikuläre Instrumentation C V/VI auf Th I/III. 

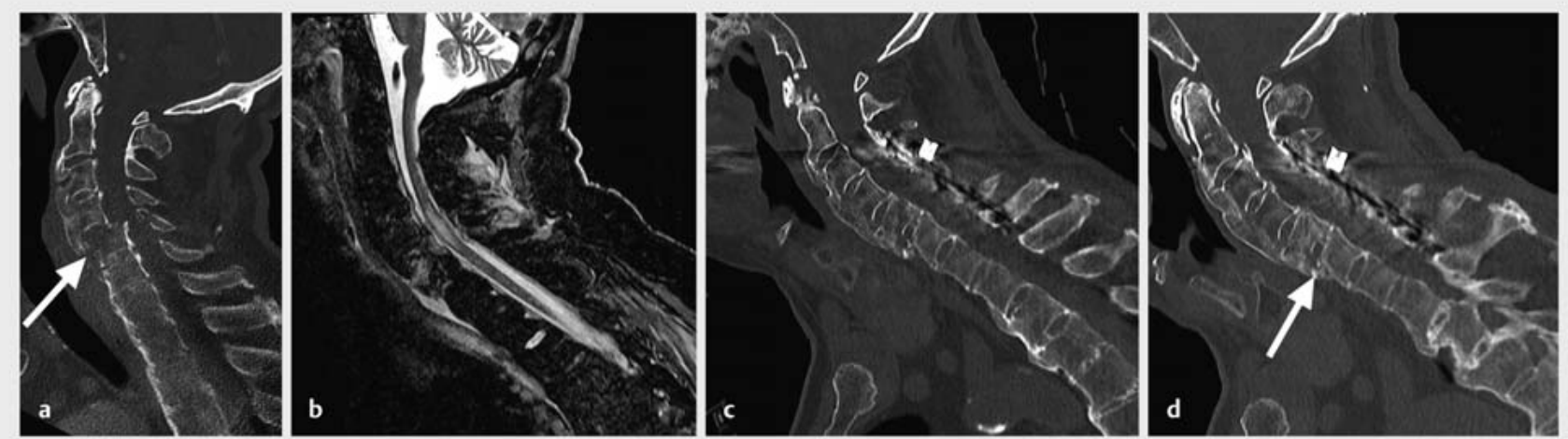

- Abb. 3 a CT der HWS, präoperatives MRT mit Myelopathiesignal auf Frakturhöhe. b Postoperative CT-Kontrolle mit anatomischer Reposition.

c CT-Kontrolle 4 Monate nach Versorgung mit guter Konsolidierungsreaktion (Pfeil), ASIA D.
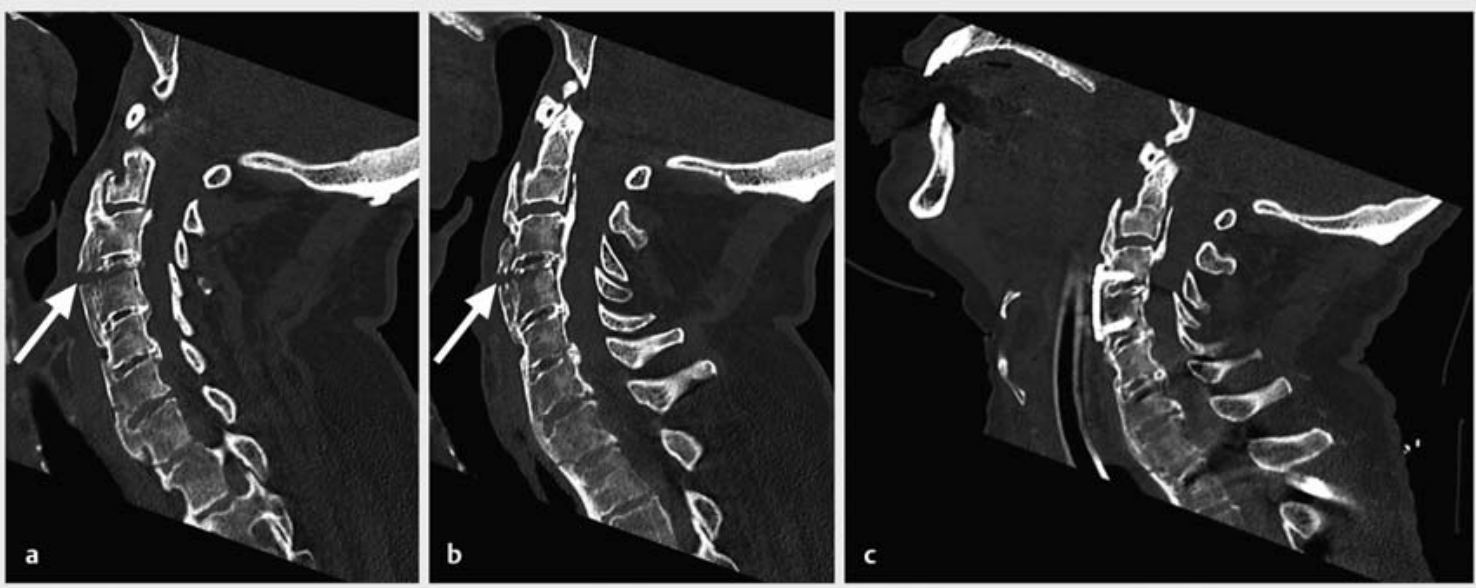

- Abb. 4 a Unfallbild (Pfeil zeigt Fraktur); b postoperatives Versorgungsbild.

Das wachsame perioperative Management bei stabiler Instrumentierung bestimmt das Outcome.

\section{Interessenkonflikt}

Der Autor gibt an, dass kein Interessenkonflikt besteht.

Autorinnen/Autoren

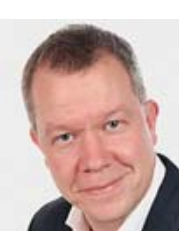

\section{Thomas Vordemvenne}

Prof. Dr. med., Thomas Vordemvenne, Chefarzt der Klinik für Unfallchirurgie und Orthopädie, Evangelisches Klinikum Bethel, Bielefeld
Korrespondenzadresse

Prof. Dr. med. Thomas Vordemvenne

Klinik für Unfallchirurgie und Orthopädie Evangelisches Klinikum Bethel - EvKB Burgsteig 13

33617 Bielefeld

thomas.vordemvenne@evkb.de

\section{Literatur}

[1] Forestier J, Rotes-Querol J. Senile ankylosing hyperostosis of the spine. Ann Rheum Dis 1950; 9: 321-330

[2] Dieterichs G, Engelken F, Link TM et al. Osteoporotic Fractures in Men (MrOS) Research Group. Diffuse idiopathic skeletal hyperostosis (DISH): relation to vertebral fractures and bone density. Osteoporosis Int 2011; 22: 1789-1797

[3] Altenbernd J, Bitu $S$, Lemburg $S$ et al. [Vertebral fractures in patients with ankylosing spondylitis: a retrospective analysis of 66 patients]. RoFo 2009; 181: 45-53 
[4] Rowed DW. Management of cervical spinal cord injury in ankylosing spondylitis: the intervertebral disc as a cause of cord compression. J Neurosurg 1992; 77: 241

[5] Smith MD, Scott JM, Murali $R$ et al. Minor neck trauma in chronic ankylosing spondylitis: a potentially fatal combination. J Clin Rheumatol 2007; 13: 81-84

[6] Heyde CE, Fakler JH, Hasenboehler E et al. Pitfalls and complications in the treatment of cervical spine fractures in patients with ankylosing spondylitis. Patient Safe Surg 2008; 2: 15

[7] Anwar F, Al-Khayer A, Allan DB et al. Delayed presentation und diagnosis of cervical spine injuries in long-standing ankylosing spondylitis. Eur Spine J 2011; 20: 403-407

[8] Löhrer L, Vieth V, Raschke MJ et al. Blunt cerebrovascular injuries in acute trauma care: a screening protocol. Eur Spine J 2012; 21: 837-843

[9] Vaccaro AR, Koerner JD, Radcliff KE et al. AOSpine subaxial cervical spine injury classification system. Eur Spine J 2016; 25: 2173-2184
[10] Taggard DA, Traynelis VC. Management of cervical spinal fractures in ankylosing spondylitis with posterior fixation. Spine 2000; 25: 2035-2039

[11] Longo UG, Loppini M, Petrillo S et al. Management of cervical fractures in ankylosing spondylitis: anterior, posterior or combined approach? Br Med Bull 2015; 115: 57-66

[12] Einsiedel T, Schmelz A, Arand M et al. Injuries of the cervical spine in patients with ankylosing spondylitis: experience at two trauma centers. J Neurosurg Spine 2006; 5: 33-45

\section{Bibliografie}

DOI https://doi.org/10.1055/s-0044-100270

OP-JOURNAL 2018; 34: 161-166 @ Georg Thieme Verlag KG Stuttgart · New York ISSN 0178-1715 